\title{
Stress Intensity Factors for a Crack in Bonded Dissimilar Materials Subjected to Various Stresses
}

\author{
K B Hamzah ${ }^{1,2}$, N M A Nik Long ${ }^{1,3, *}$, N Senu ${ }^{1,3}$, Z K Eshkuvatov ${ }^{4}$, M R Ilias ${ }^{5}$ \\ ${ }^{1}$ Laboratory of Computational Sciences and Mathematical Physics, Institute for Mathematical Research, \\ University Putra Malaysia, Malaysia \\ ${ }^{2}$ Fakulti Teknologi Kejuruteraan Mekanikal dan Pembuatan, Technical University of Malaysia Malacca, Malaysia \\ ${ }^{3}$ Department of Mathematics, Faculty of Science, University Putra Malaysia, Malaysia \\ ${ }^{4}$ Faculty of Science and Technology, University Putra Malaysia, Malaysia \\ ${ }^{5}$ Department of Mathematical Sciences, Faculty of Computer and Mathematical Sciences, Universiti Teknologi MARA, Malaysia
}

Copyright $\bigcirc 2019$ by authors, all rights reserved. Authors agree that this article remains permanently open access under the terms of the Creative Commons Attribution License 4.0 International License

\begin{abstract}
The modified complex variable function method with the continuity conditions of the resultant force and displacement function are used to formulate the hypersingular integral equations (HSIE) for an inclined crack and a circular arc crack lies in the upper part of bonded dissimilar materials subjected to various remote stresses. The curve length coordinate method and appropriate quadrature formulas are used to solve numerically the unknown crack opening displacement (COD) function and the traction along the crack as the right hand term of HSIE. The obtained COD is then used to compute the stress intensity factors (SIF), which control the stability behavior of bodies or materials containing cracks or flaws. Numerical results showed the behavior of the nondimensional SIF at the crack tips. It is observed that the nondimensional SIF at the crack tips depend on the various remote stresses, the elastic constants ratio, the crack geometries and the distance between the crack and the boundary.
\end{abstract}

Keywords Stress Intensity Factor, Bonded Dissimilar Materials, Complex Variable Function, Hypersingular Integral Equation

\section{Introduction}

A number of papers have been publish to analyze the behaviour of stress intensity factors (SIF) at the crack tips subjected to specific remote stress for the crack problems in an infinite plane [1,2], finite plane [3,4], half plane $[5,6]$ or bonded dissimilar materials [7-9]. The body force method with continuous distributions along cracks were used to find the nondimensional SIF of the crack problems in bonded dissimilar materials subjected to various stress
[7]. The Fredholm integral equations with density distributions as undetermined functions were used to calculate the nondimensional SIF for multiple crack problems in bonded dissimilar materials [8]. The logarithmic singular integral equations were used to solve the nondimensional SIF for a circular arc crack lie in the upper part of bonded dissimilar materials [9]. The combinations of Chebyshev polynomials and collocation methods were utilized to solve the nondimensional SIF of a perpendicular crack to the interface of bonded dissimilar materials [10]. The effect of elastic constants ratio to the penny-shaped crack problems in bonded dissimilar materials was determined by reducing the dual integral equations to an infinite system of simultaneous equations [11]. The solidification crack growth patterns in bonded dissimilar materials were predicted by analyzes the nondimensional SIF [12]. The nondimensional SIF of an interface crack in bonded dissimilar materials was evaluated by combining the extended finite element method and a domain independent interaction integral method [13].

The objectives of this paper is to formulate the HSIE and analyze the behavior of nondimensional SIF by using the modified complex variable function method for an inclined and a curved crack lie in the upper part of bonded dissimilar materials subjected to the various remote stresses such as shear stress $\sigma x_{1}=\sigma x_{2}=p$, normal stress $\sigma y_{1}=\sigma y_{2}=p$, tearing stress $\sigma x_{1} y_{1}=\sigma x_{2} y_{2}=p$ or mixed stress $\sigma x_{1}=\sigma x_{2}=\sigma y_{1}=\sigma y_{2}=p$.

\section{Mathematical Formulation}

The complex variable function method plays an important role in solving the cracks problem in plane elasticity [14]. In this method, the stress components 
$\left(\sigma_{x}, \sigma_{y}, \sigma_{x y}\right)$, resultant force function $f(X, Y)$ and displacements $(u, v)$ are related to the two complex potentials $\phi(z)$ and $\omega(z)$ as follows

$$
\begin{gathered}
\sigma_{y}-i \sigma_{x y}=\phi^{\prime}(z)+(z-\bar{z}) \overline{\phi^{\prime \prime}(z)}+\omega^{\prime}(\bar{z}) \\
f=-Y+i X=\phi(z)+(z-\bar{z}) \overline{\phi^{\prime \prime}(z)}+\omega(\bar{z}) \\
2 G(u+i v)=\kappa \phi(z)-(z-\bar{z}) \overline{\phi^{\prime}(z)}-\omega(\bar{z})
\end{gathered}
$$

where $G$ is shear modulus of elasticity, $\kappa=(3-v) /(1+v)$ for plane stress, $\kappa=3-4 v$ for plane strain and $\mathrm{v}$ is Poisson's ratio. The function $\omega(z)$ defined as

$$
\omega(z)=z \overline{\phi^{\prime}}(z)+\bar{\psi}(z)
$$

where an analytic function $\bar{\phi}(z)$ defined as $\bar{\phi}(z)=\overline{\phi(\bar{z})}$. The derivative in a specified direction (2) and apply (4), yields

$$
\begin{aligned}
& \frac{d}{d z}\{-Y+i X\}=\phi^{\prime}(z)+(z-\bar{z}) \overline{\phi^{\prime \prime \prime}(z)} \frac{d \bar{z}}{d z}+\omega^{\prime}(\bar{z}) \frac{d \bar{z}}{d z} \\
& =\phi^{\prime}(z)+\overline{\phi^{\prime}(z)}+\frac{d \bar{z}}{d z}\left[z \overline{\phi^{\prime \prime}(z)}+\overline{\psi^{\prime}(z)}\right]=N+i T
\end{aligned}
$$

where $N+i T$ denotes the normal and tangential traction along the crack segment $\overline{z, z+d z}$. The complex potentials for a crack $L$ in an infinite plane can be expressed by

$$
\phi(z)=\frac{1}{2 \pi} \int_{L} \frac{g(t) d t}{t-z},
$$

$$
\psi(z)=\frac{1}{2 \pi} \int_{L} \frac{g(t) d \bar{t}}{t-z}+\frac{1}{2 \pi} \int_{L} \frac{\overline{g(t)} d t}{t-z}-\frac{1}{2 \pi} \int_{L} \frac{\bar{t} g(t) d t}{(t-z)^{2}}
$$

where $g(t)$ is crack opening displacement (COD) function defined by

$$
g(t)=\frac{2 G}{i(\kappa+1)}\left[(u(t)+i v(t))^{+}-(u(t)+i v(t))^{-}\right]
$$

$(u(t)+i v(t))^{+} \quad$ and $\quad(u(t)+i v(t))^{-} \quad$ denote the displacements at point $t$ of the upper and lower crack faces, respectively [15].

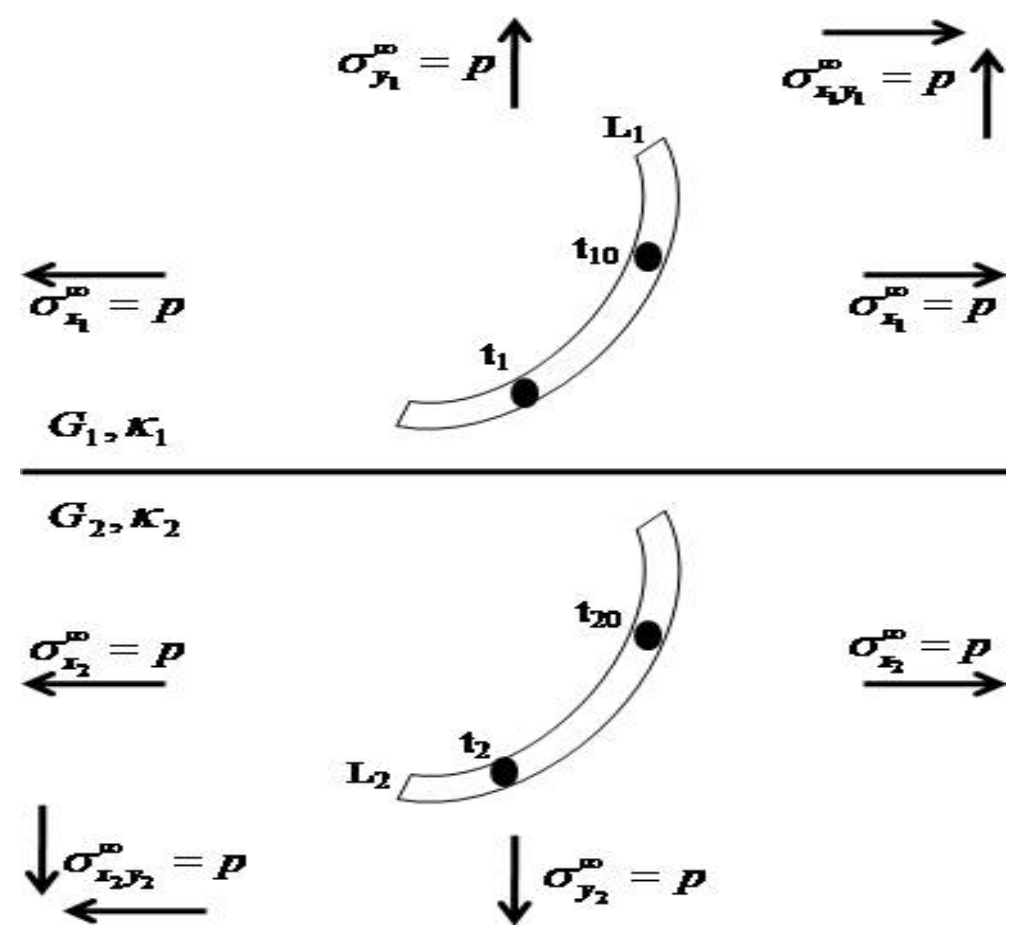

Figure 1. Two cracks $L_{1}$ and $L_{2}$ in bonded dissimilar materials 
Consider two cracks lie in the upper and lower parts of bonded dissimilar materials subjected to various stresses defined in Figure 1. The conditions for remote stresses in bonded dissimilar materials are

$$
\varepsilon_{x_{1}}=\varepsilon_{x_{2}}, \varepsilon_{y_{1}}=\varepsilon_{y_{2}}, \varepsilon_{x_{1} y_{1}}=\varepsilon_{x_{2} y_{2}}, \varepsilon_{x_{1}}=\varepsilon_{x_{2}}=\varepsilon_{y_{1}}=\varepsilon_{y_{2}}
$$

where subscripts 1 and 2 are the stress component for the upper and lower parts of bonded dissimilar materials, respectively. Those stresses can be expresses as

$$
\begin{gathered}
\varepsilon_{x_{1}}=\frac{1}{E_{1}}\left(\sigma_{x_{1}}-v_{1} \sigma_{y_{1}}\right), \varepsilon_{x_{2}}=\frac{1}{E_{2}}\left(\sigma_{x_{2}}-v_{2} \sigma_{y_{2}}\right) \\
\varepsilon_{y_{1}}=\frac{1}{E_{1}}\left(\sigma_{y_{1}}-v_{1} \sigma_{x_{1}}\right), \varepsilon_{y_{2}}=\frac{1}{E_{2}}\left(\sigma_{y_{2}}-v_{2} \sigma_{x_{2}}\right) \\
\varepsilon_{x_{1} y_{1}}=\frac{1+v_{1}}{E_{1}} \sigma_{x_{1} y_{1}}, \varepsilon_{x_{2} y_{2}}=\frac{1+v_{2}}{E_{2}} \sigma_{x_{2} y_{2}} \\
\varepsilon_{x_{1}}=\varepsilon_{y_{1}}=\frac{1-v_{1}}{E_{1}} \sigma_{x_{1}}=\frac{1-v_{1}}{E_{1}} \sigma_{y_{1}}, \\
\varepsilon_{x_{2}}=\varepsilon_{y_{2}}=\frac{1-v_{2}}{E_{2}} \sigma_{x_{2}}=\frac{1-v_{2}}{E_{2}} \sigma_{y_{2}}
\end{gathered}
$$

where $E_{1}=2 G_{1}\left(1+v_{1}\right)$ and $E_{2}=2 G_{2}\left(1+v_{2}\right)$ are Young's modulus of elasticity for upper and lower parts of bonded dissimilar materials, respectively. For the shear stress $\sigma x_{1}=\sigma x_{2}=p$, normal stress $\sigma y_{1}=\sigma y_{2}=p$, tearing stress $\sigma x_{1} y_{1}=\sigma x_{2} y_{2}=p$ and mixed stress $\sigma x_{1}=\sigma x_{2}=\sigma y_{1}=\sigma y_{2}=p$ occur when the others stresses does not exist, then (10), (11), (12), (13) reduced to

$$
\begin{gathered}
\frac{1}{E_{1}} \sigma_{x_{1}}=\frac{1}{E_{2}} \sigma_{x_{2}} \\
\frac{1}{E_{1}} \sigma_{y_{1}}=\frac{1}{E_{2}} \sigma_{y_{2}} \\
\frac{1+v_{1}}{E_{1}} \sigma_{x_{1} y_{1}}=\frac{1+v_{2}}{E_{2}} \sigma_{x_{2} y_{2}} \\
\frac{1-v_{1}}{E_{1}} \sigma_{x_{1}}=\frac{1-v_{2}}{E_{2}} \sigma_{x_{2}} \text { or } \frac{1-v_{1}}{E_{1}} \sigma_{y_{1}}=\frac{1-v_{2}}{E_{2}} \sigma_{y_{2}}
\end{gathered}
$$

respectively.

The modified complex potentials for the crack lies in the upper part of bonded dissimilar materials are defined as

$$
\phi_{1}(z)=\phi_{1 p}(z)+\phi_{1 c}(z), \psi_{1}(z)=\psi_{1 p}(z)+\psi_{1 c}(z)
$$

where subscript $p$ and $c$ represent the principal and complementary parts, respectively. Whereas, for the crack lies in the lower part, the complex potentials are represented by $\phi_{2}(z)$ and $\psi_{2}(z)$. The continuity conditions for the resultant force (2) and displacement functions (3) by using (4), yields

$$
\begin{aligned}
& {\left[\phi_{1}(t)+t \overline{\phi_{1}{ }^{\prime}(t)}+\overline{\psi_{1}(t)}\right]^{+}=\left[\phi_{2}(t)+t \overline{\phi_{2}{ }^{\prime}(t)}+\overline{\psi_{2}(t)}\right]^{-}} \\
& G_{2}\left[\kappa_{1} \phi_{1}(t)-t \overline{\phi_{1}{ }^{\prime}(t)}-\overline{\psi_{1}(t)}\right]^{+}=G_{1}\left[\kappa_{2} \phi_{2}(t)-t \overline{\phi_{2}{ }^{\prime}(t)}-\overline{\psi_{2}(t)}\right]^{-}
\end{aligned}
$$

where $t \in L$. Applying (18) into (19) and (20), we can obtained the following complex potentials

$$
\begin{gathered}
\phi_{1 c}(z)=\frac{G_{2}-G_{1}}{G_{1}+\kappa_{1} G_{2}}\left[z \overline{\phi_{1 p}^{\prime}}(z)+\overline{\psi_{1 p}}(z)\right], z \in S_{1}+L_{b} \\
\psi_{1 c}(z)=\frac{\kappa_{1} G_{2}-\kappa_{2} G_{1}}{G_{2}+\kappa_{2} G_{1}} \overline{\phi_{1 p}}(z)-\frac{G_{2}-G_{1}}{G_{1}+\kappa_{1} G_{2}}\left[z \overline{\phi_{1 p}^{\prime}}(z)\right. \\
\left.+z^{2} \overline{\phi_{1 p}^{\prime \prime}}(z)+z \overline{\psi_{1 p}^{\prime}}(z)\right], z \in S_{1}+L_{b} \\
\phi_{2}(z)=\left(1+\frac{\kappa_{1} G_{2}-\kappa_{2} G_{1}}{G_{2}+\kappa_{2} G_{1}}\right) \phi_{1 p}(z), z \in S_{2}+L_{b} \\
\psi_{2}(z)=\left(\frac{G_{2}-G_{1}}{G_{1}+\kappa_{1} G_{2}}-\frac{\kappa_{1} G_{2}-\kappa_{2} G_{1}}{G_{2}+\kappa_{2} G_{1}}\right) z \phi_{1 p}^{\prime}(z) \\
+\left(1+\frac{G_{2}-G_{1}}{G_{1}+\kappa_{1} G_{2}}\right) \psi_{1 p}(z), z \in S_{2}+L_{b}
\end{gathered}
$$

where $L_{b}$ is boundary of two bonded half materials, $S_{l}$ and $S_{2}$ are upper and lower parts of bonded dissimilar materials, respectively, and the principle part of complex potentials are referred to an infinite materials.

The HSIE for a crack lies in the upper part of bonded dissimilar materials can be obtained by substituted (18) into (5) and applying (6), (7), (21) and (22), then letting point $z$ approaches $t_{0}$ on the crack and changing $d \bar{z} / d z$ into $d \bar{t}_{0} / d t_{0}$, yields

$$
\begin{aligned}
N\left(t_{0}\right)+ & i T\left(t_{0}\right)=\frac{1}{\pi} h p \int_{L} \frac{g(t) d t}{\left(t-t_{0}\right)^{2}} \\
& +\frac{1}{2 \pi} \int_{L} A_{1}\left(t, t_{0}\right) g(t) d t+\frac{1}{2 \pi} \int_{L} A_{2}\left(t, t_{0}\right) \overline{g(t)} d t
\end{aligned}
$$

where

$$
\begin{aligned}
A_{1}\left(t, t_{0}\right)= & B_{1}\left(t, t_{0}\right)+\frac{G_{2}-G_{1}}{G_{1}+\kappa_{1} G_{2}}\left[\overline{B_{3}\left(t, t_{0}\right)}-\frac{d \overline{t_{0}}}{d t_{0}}\left(B_{5}\left(t, t_{0}\right)\right.\right. \\
& \left.+\overline{B_{4}\left(t, t_{10}\right)}\right)+\frac{d \bar{t}}{d t}\left(B_{4}\left(t, t_{0}\right)+\overline{B_{4}\left(t, t_{0}\right)}\right) \\
& \left.-\frac{d \bar{t}}{d t} \frac{d \bar{t}_{0}}{d t_{0}} B_{6}\left(t, t_{0}\right)\right]+\frac{\kappa_{1} G_{2}-\kappa_{2} G_{1}}{G_{2}+\kappa_{2} G_{1}} \frac{\overline{d t_{0}}}{d t_{0}} \overline{B_{4}\left(t, t_{0}\right)} \\
A_{2}\left(t, t_{0}\right)= & B_{2}\left(t, t_{0}\right)+\frac{G_{2}-G_{1}}{G_{1}+\kappa_{1} G_{2}}\left[B_{4}\left(t, t_{0}\right)+\overline{B_{4}\left(t, t_{0}\right)}\right. \\
& \left.-\frac{d \bar{t}_{0}}{d t_{0}} B_{6}\left(t, t_{0}\right)+\frac{d \bar{t}}{d t} B_{3}\left(t, t_{0}\right)\right]
\end{aligned}
$$


and

$$
\begin{aligned}
& B_{1}\left(t, t_{0}\right)=\frac{1}{\left(t-t_{0}\right)^{2}}\left[\frac{\left(t-t_{0}\right)^{2}}{\left(\bar{t}-\bar{t}_{0}\right)^{2}} \frac{d \bar{t}}{d t} \frac{d \bar{t}_{0}}{d t_{0}}-1\right] \\
& B_{2}\left(t, t_{0}\right)=\frac{t-t_{0}}{\left(\bar{t}-\bar{t}_{0}\right)^{3}}\left[\frac{\left(\bar{t}-\bar{t}_{0}\right)}{\left(t-t_{0}\right)}\left[\frac{d \bar{t}}{d t}+\frac{d \bar{t}_{0}}{d t_{0}}\right)-2 \frac{d \bar{t}}{d t} \frac{d \bar{t}_{0}}{d t_{0}}\right] \\
& B_{3}\left(t, t_{0}\right)=\frac{1}{\left(\bar{t}-t_{0}\right)^{2}}+\frac{2\left(t_{0}-t\right)}{\left(\bar{t}-t_{0}\right)^{3}} \\
& B_{4}\left(t, t_{0}\right)=\frac{1}{\left(\bar{t}-t_{0}\right)^{2}} \\
& B_{5}\left(t, t_{0}\right)=\frac{2\left(3 \bar{t}_{0}-2 t_{0}-\bar{t}\right)}{\left(t-\bar{t}_{0}\right)^{3}}+\frac{6\left(\bar{t}_{0}-\bar{t}^{3}\right)\left(\bar{t}_{0}-t_{0}\right)}{\left(t-\bar{t}_{0}\right)^{4}} \\
& B_{6}\left(t, t_{0}\right)=\frac{1}{\left(t-\bar{t}_{0}\right)^{2}}+\frac{\left(t-\bar{t}_{0}\right)^{3}}{(t} .
\end{aligned}
$$

Note that if $G_{2}=0$ and $G_{1}=G_{2}$, then the HSIE for a crack in bonded dissimilar (25) reduces to the HSIE for a crack in the half plane [5] and in an infinite plane elasticity [2], respectively.

It is well known that to solve the HSIE (25), the curved length coordinate method is used which transform the COD function $g(t)$ to the square root singularity $\sqrt{a^{2}-s^{2}}$ as follows $[5,15]$

$$
H(s)=\frac{g(t)}{\sqrt{a^{2}-s^{2}}}
$$

with the following quadrature formulas [16]

$$
\begin{gathered}
\frac{1}{\pi} \int_{-a}^{a} \frac{\sqrt{a^{2}-s^{2}} H(s) d s}{\left(s-s_{0}\right)^{2}}=\sum_{j=1}^{M+1} W_{j}\left(s_{0}\right) H\left(s_{j}\right) \\
\frac{1}{\pi} \int_{-a}^{a} \sqrt{a^{2}-s^{2}} H(s) d s=\frac{1}{M+2} \sum_{j=1}^{M+1}\left(a^{2}-s_{0}^{2}\right) H\left(s_{j}\right)
\end{gathered}
$$

where $H(s)$ is a given function, $M \in \mathbb{Z}^{+}$,

$$
s_{j}=a \cos \left(\frac{j \pi}{M+2}\right), j=1,2, \ldots, M+1
$$

and

and

$$
W_{j}\left(s_{0}\right)=-\frac{2}{M+2} \sum_{n=0}^{M}(n+1) \sin \left(\frac{j \pi}{M+2}\right) \sin \left(\frac{(n+1) j \pi}{M+2}\right) U_{n}\left(\frac{s_{j 0}}{a}\right)
$$

and the observation points

$$
s_{0}=s_{0, k}=a \cos \left(\frac{k \pi}{M+2}\right), k=1,2, \ldots, M+1 .
$$

Here $U_{n}(t)$ is a Chebyshev polynomial of the second kind, define by

$$
U_{n}(t)=\frac{\sin ((n+1) \theta)}{\sin \theta}, \text { where } t=\cos \theta .
$$

The normal and tangential components $N+i T$ of the HSIE for the shear stress $\sigma x_{1}=\sigma x_{2}=p$, normal stress $\sigma y_{1}=\sigma y_{2}=p$, tearing stress $\sigma x_{1} y_{1}=\sigma x_{2} y_{2}=p$ and mixed stress $\sigma x_{1}=\sigma x_{2}=\sigma y_{1}=\sigma y_{2}=p$ with an angle of the crack $\alpha$ are defined as follows

$$
\begin{gathered}
N+i T=-p \sin ^{2} \alpha-i p \sin \alpha \cos \alpha \\
N+i T=-p \cos ^{2} \alpha+i p \sin \alpha \cos \alpha \\
N+i T=2 p \sin \alpha \cos \alpha+i p\left(\cos ^{2} \alpha-\sin ^{2} \alpha\right) \\
N+i T=-p+i 0 .
\end{gathered}
$$

\section{Results and Discussions}

Stress intensity factor (SIF) at crack tips $A_{j}$ is defined as

$$
\left(K_{1}-i K_{2}\right)_{A_{j}}=\sqrt{2 \pi} \lim _{t \rightarrow t_{A_{j}}} \sqrt{\left|t-t_{A_{j}}\right|} g^{\prime}(t), j=1,2 .
$$

Consider an inclined crack lie in the upper part of bonded dissimilar materials subjected to various stresses as defined in Figure 2.

Table 1 displays the nondimensional SIF versus $h / 2 R$ at the crack tip subjected to tearing stress $\sigma x_{1} y_{1}=\sigma x_{2} y_{2}=p$ when with $\alpha=0^{\circ}$ different values of elastic constant ratio $G_{2} / G_{1}$ where $R$ and $h$ are defined as in Figure 2. Our results are totally agrees with those of Isida and Noguchi [7]. It is observed that the nondimensional SIF at $F_{1 A l}$ and $F_{2 A l}$ are equals to negative of $F_{1 A 2}$ and $F_{2 A 2}$, respectively. 


$$
\sigma_{\boldsymbol{y}_{1}}^{\mathbf{m}}=\boldsymbol{p} \uparrow
$$
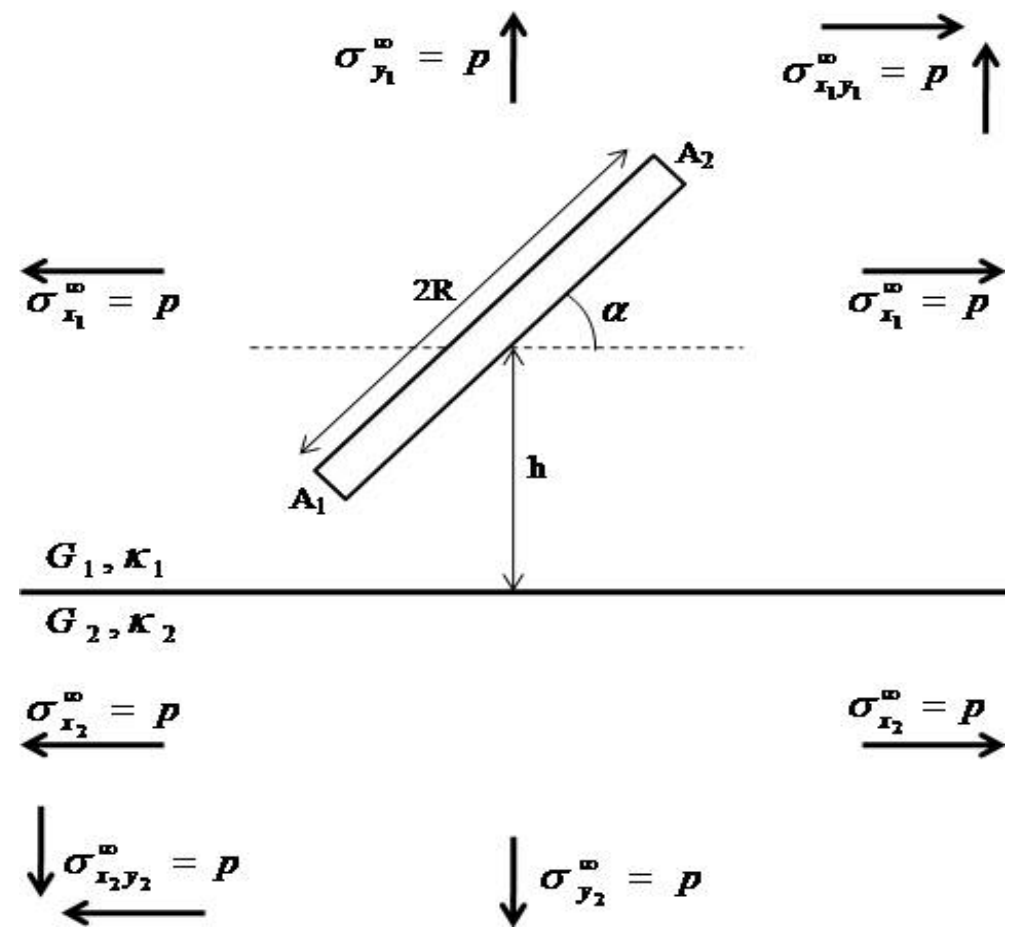

Figure 2. An inclined crack in bonded dissimilar materials

Table 1. Nondimensional SIF for an inclined crack when $\alpha=0^{o}$ subjected to $\sigma_{x_{1} y_{1}}=\sigma_{x_{2} y_{2}}=p$

\begin{tabular}{|c|c|c|c|c|c|c|c|c|c|c|}
\hline \multirow{2}{*}{$\mathrm{G}_{2} / \mathrm{G}_{1}$} & \multirow{2}{*}{ SIF } & \multicolumn{9}{|c|}{$\mathrm{h} / 2 \mathrm{R}$} \\
\hline & & 0.1 & 0.2 & 0.3 & 0.4 & 0.5 & 0.6 & 0.7 & 0.8 & 0.9 \\
\hline \multirow{4}{*}{0.0} & $\mathrm{~F}_{1 \mathrm{~A} 1} *$ & 0.6151 & 0.3876 & 0.2639 & 0.1854 & 0.1331 & 0.0973 & 0.0724 & 0.0548 & 0.0422 \\
\hline & $\mathrm{F}_{1 \mathrm{~A} 1} * *$ & 0.6150 & 0.3880 & 0.2640 & 0.1850 & 0.1330 & 0.0970 & 0.0720 & 0.0550 & 0.0420 \\
\hline & $\mathrm{F}_{2 \mathrm{~A} 1} *$ & 1.2077 & 1.1329 & 1.1126 & 1.0993 & 1.0874 & 1.0762 & 1.0661 & 1.0573 & 1.0497 \\
\hline & $\mathrm{F}_{2 \mathrm{~A} 1} * *$ & 1.2080 & 1.1330 & 1.1130 & 1.0990 & 1.0870 & 1.0760 & 1.0660 & 1.0570 & 1.0500 \\
\hline \multirow{4}{*}{0.5} & $\mathrm{~F}_{1 \mathrm{~A} 1} *$ & 0.0878 & 0.0658 & 0.0516 & 0.0398 & 0.0303 & 0.0230 & 0.0175 & 0.0135 & 0.0105 \\
\hline & $\mathrm{F}_{1 \mathrm{~A} 1} * *$ & 0.0880 & 0.0660 & 0.0520 & 0.0400 & 0.0300 & 0.0230 & 0.0170 & 0.0130 & 0.0100 \\
\hline & $\mathrm{F}_{2 \mathrm{~A} 1} *$ & 1.0864 & 1.0512 & 1.0372 & 1.0303 & 1.0257 & 1.0221 & 1.0190 & 1.0164 & 1.0142 \\
\hline & $\mathrm{F}_{2 \mathrm{~A} 1} * *$ & 1.0860 & 1.0510 & 1.0370 & 1.0300 & 1.0260 & 1.0220 & 1.0190 & 1.0160 & 1.0140 \\
\hline \multirow{4}{*}{2.0} & $\mathrm{~F}_{1 \mathrm{~A} 1} *$ & -0.0696 & -0.0514 & -0.0403 & -0.0316 & -0.0245 & -0.0188 & -0.0145 & -0.0112 & -0.0087 \\
\hline & $\mathrm{F}_{1 \mathrm{A1} 1} * *$ & -0.0700 & -0.0510 & -0.0400 & -0.0320 & -0.0240 & -0.0190 & -0.0140 & -0.0110 & -0.0090 \\
\hline & $\mathrm{F}_{2 \mathrm{~A} 1} *$ & 0.9176 & 0.9456 & 0.9602 & 0.9684 & 0.9738 & 0.9778 & 0.9811 & 0.9838 & 0.9861 \\
\hline & $\mathrm{F}_{2 \mathrm{~A} 1} * *$ & 0.9180 & 0.9460 & 0.9600 & 0.9680 & 0.9740 & 0.9780 & 0.9810 & 0.9840 & 0.9860 \\
\hline
\end{tabular}

*Present study

**Isida and Noguchi [7] 
The Figures 3 and 4 present the nondimensional SIF for an inclined crack lies in upper part of bonded dissimilar materials subjected to various stresses at crack tips $A_{l}$ and $A_{2}$, respectively, when $R / h=0.9$ and $\alpha$ varies (Figure 2). It is found that as $\alpha$ increases the nondimensional SIF increases subjected to shear stress $\sigma x_{1}=\sigma x_{2}=p$ and decreases subjected to normal stress $\sigma_{y_{1}}=\sigma_{y_{2}}=p$ at crack tips $A_{1}$ and $A_{2}$. For tearing stress $\sigma x_{1} y_{1}=\sigma x_{2} y_{2}=p$ the nondimensional SIF increases when $\alpha>50^{\circ}$ at crack tips $A_{1}$ and $A_{2}$. Whereas as $\alpha$ increases the nondimensional SIF at crack tips $A_{1}$ and $A_{2}$ does not show any significant differences for $G_{2} / G_{1}>0.0$ subjected to mixed stress $\sigma x_{1}=\sigma x_{2}=\sigma y_{1}=\sigma y_{2}=p$. The nondimensional SIF decreases as $G_{2} / G_{1}$ increases subjected to shear stress $\sigma x_{1}=\sigma x_{2}=p$, normal stress $\sigma y_{1}=\sigma y_{2}=p$ and mixed stress $\sigma x_{1}=\sigma x_{2}=\sigma y_{1}=\sigma y_{2}=p$ at crack tips $\mathrm{A}_{1}$ and $\mathrm{A}_{2}$. However for tearing stress $\sigma x_{1} y_{1}=\sigma x_{2} y_{2}=p$ the nondimensional SIF increases as $G_{2} / G_{1}$ increases at crack tips $A_{1}$ and $A_{2}$.

Consider a circular arc crack lie in the upper part of bonded dissimilar materials subjected to various stresses as defined in Figure 5.

Figure 6 shows the nondimensional SIF for a circular arc crack lies in the upper part of bonded dissimilar materials subjected to various stresses at crack tip $A_{l}$ when $h=0.5 R$ and $\alpha$ varies as defined in Figure 5. It is observed that the nondimensional SIF at crack $A_{1}$ equal to the SIF $A_{2}$ subjected to shear stress $\sigma x_{1}=\sigma x_{2}=p$, normal stress $\sigma y_{1}=\sigma y_{2}=p$ and mixed stress $\sigma x_{1}=\sigma x_{2}=\sigma y_{1}=\sigma y_{2}=p$. Whereas for tearing stress $\sigma x_{1} y_{1}=\sigma x_{2} y_{2}=p$ the nondimensional SIF at crack $A_{l}$ equal to the negative SIF at $A_{2}$. The nondimensional SIF increases subjected to shear stress $\sigma x_{1}=\sigma x_{2}=p$ and tearing stress $\sigma x_{1} y_{1}=\sigma x_{2} y_{2}=p$, however SIF decreases subjected to normal stress $\sigma y_{1}=\sigma y_{2}=p$ and mixed stress $\sigma x_{1}=\sigma x_{2}=\sigma y_{1}=\sigma y_{2}=p$ as $\alpha$ increases at crack tip $\mathrm{A}_{1}$.
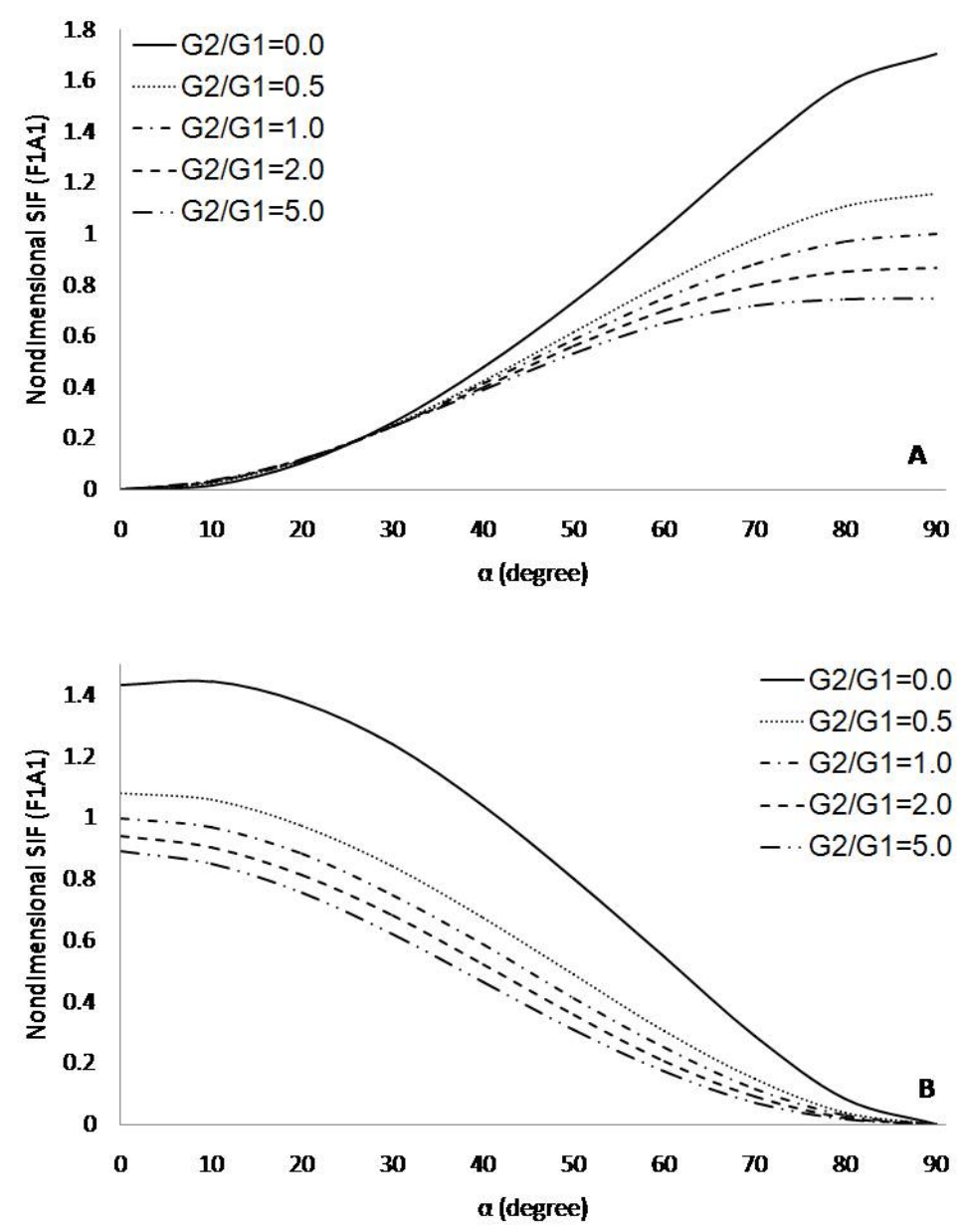

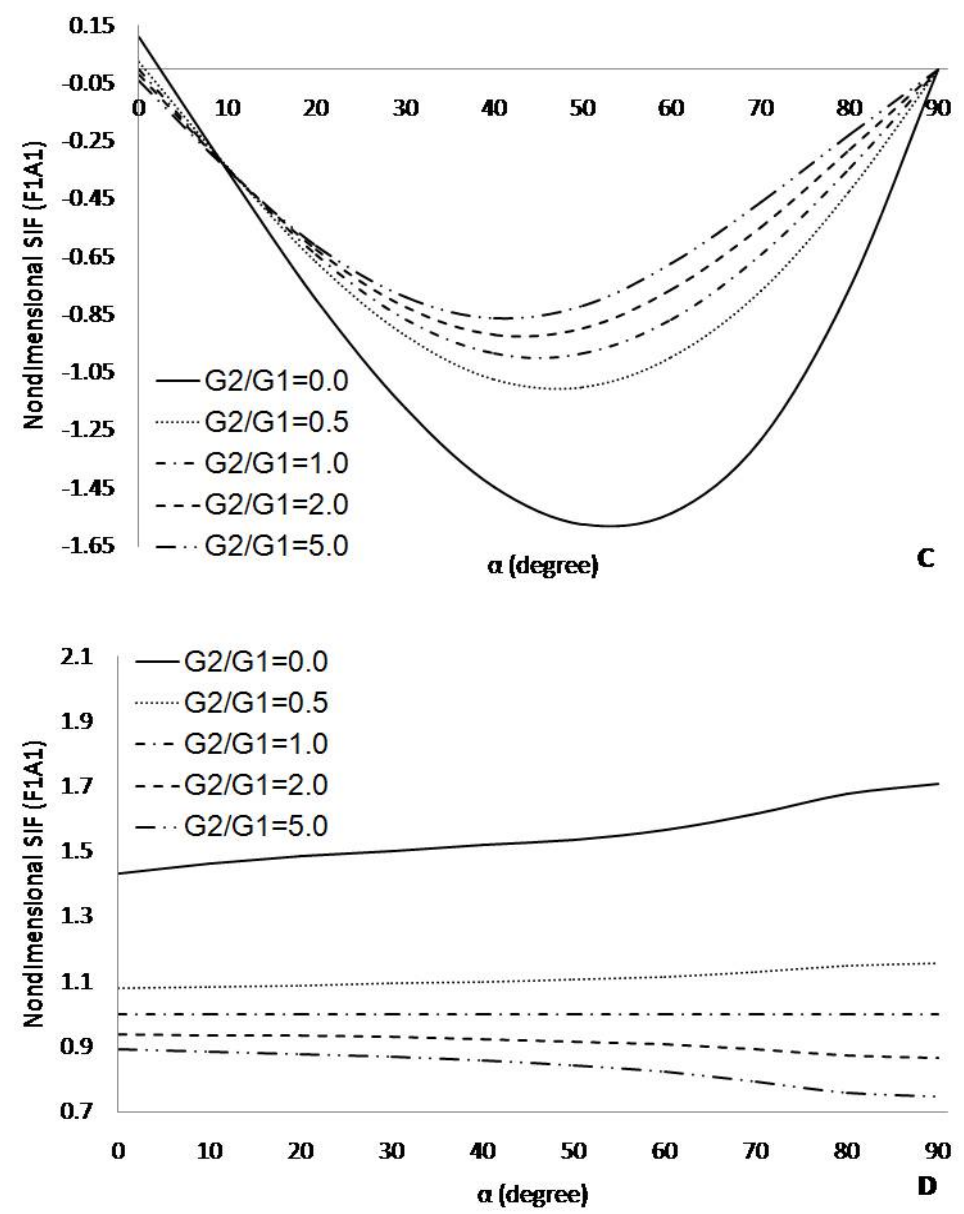

Figure 3. Nondimensional SIF versus $\alpha$ when $R / h=0.9$ at crack tip $A_{l}$ subject to: Part A Shear stress, Part B Normal stress, Part C Tearing Stress and Part D Mixed stress

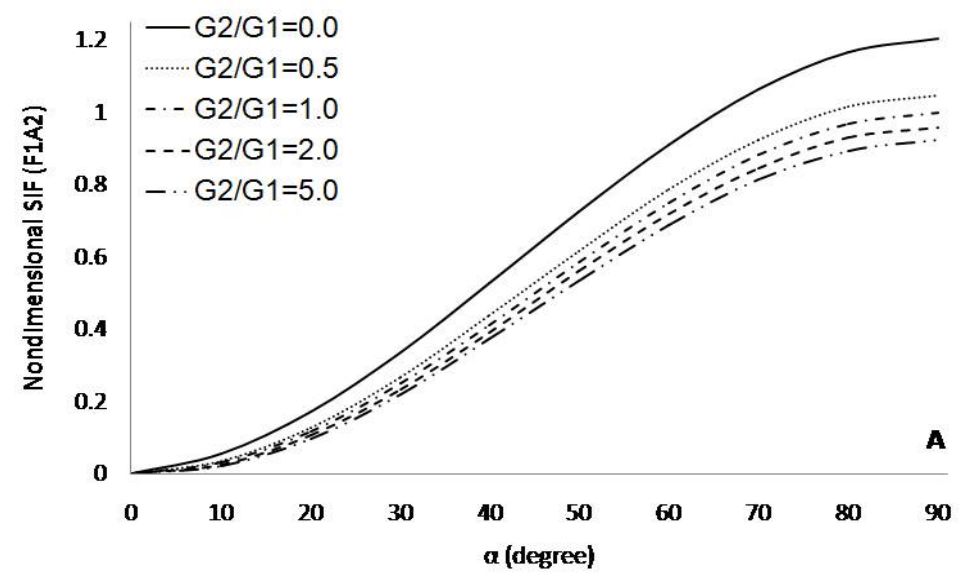



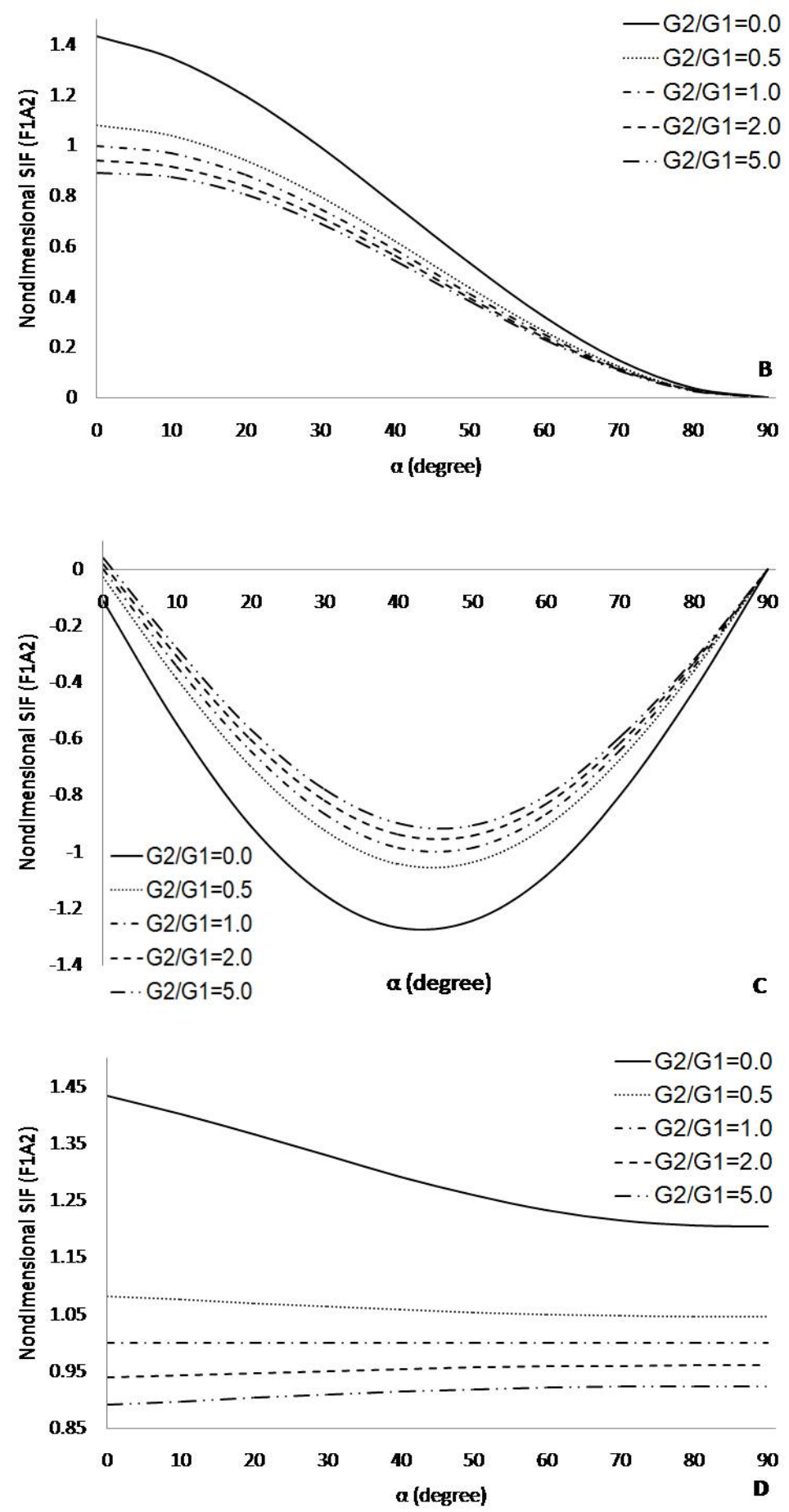

Figure 4. Nondimensional SIF versus $\alpha$ when $R / h=0.9$ at crack tip $A_{2}$ subject to: Part A Shear stress, Part B Normal stress, Part C Tearing Stress and Part D Mixed stress 


$$
\sigma_{y_{1}}^{\mathbf{m}}=p \uparrow
$$

$$
\overrightarrow{\sigma_{x_{1} y_{1}}^{m}=p} \uparrow
$$
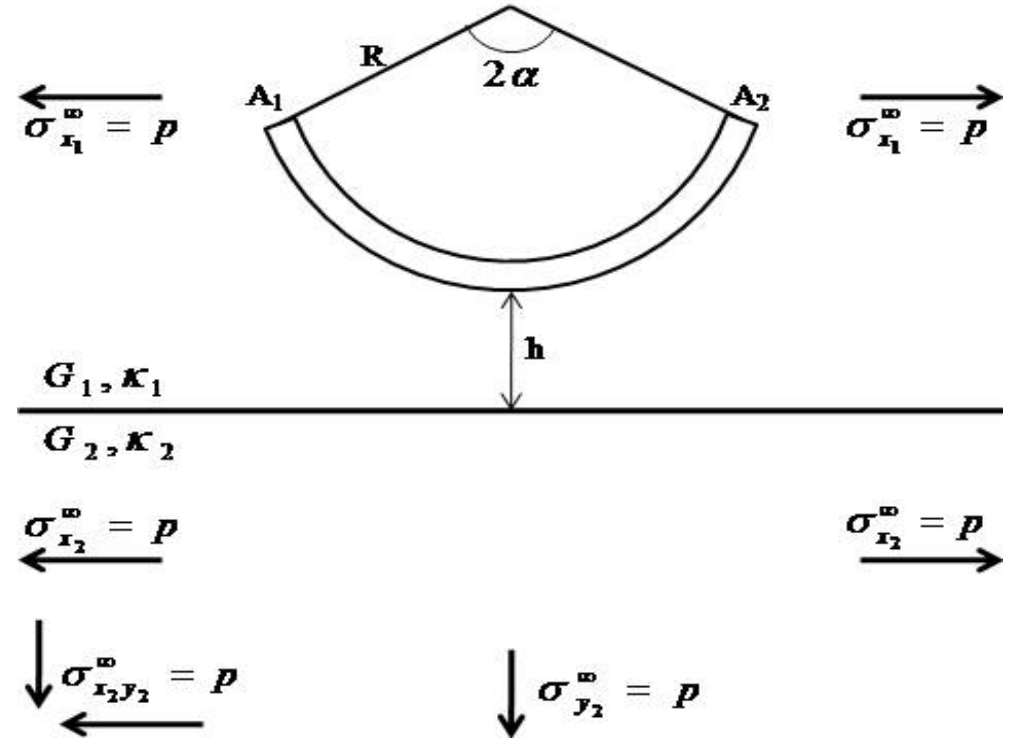

Figure 5. A circular arc crack in bonded dissimilar materials
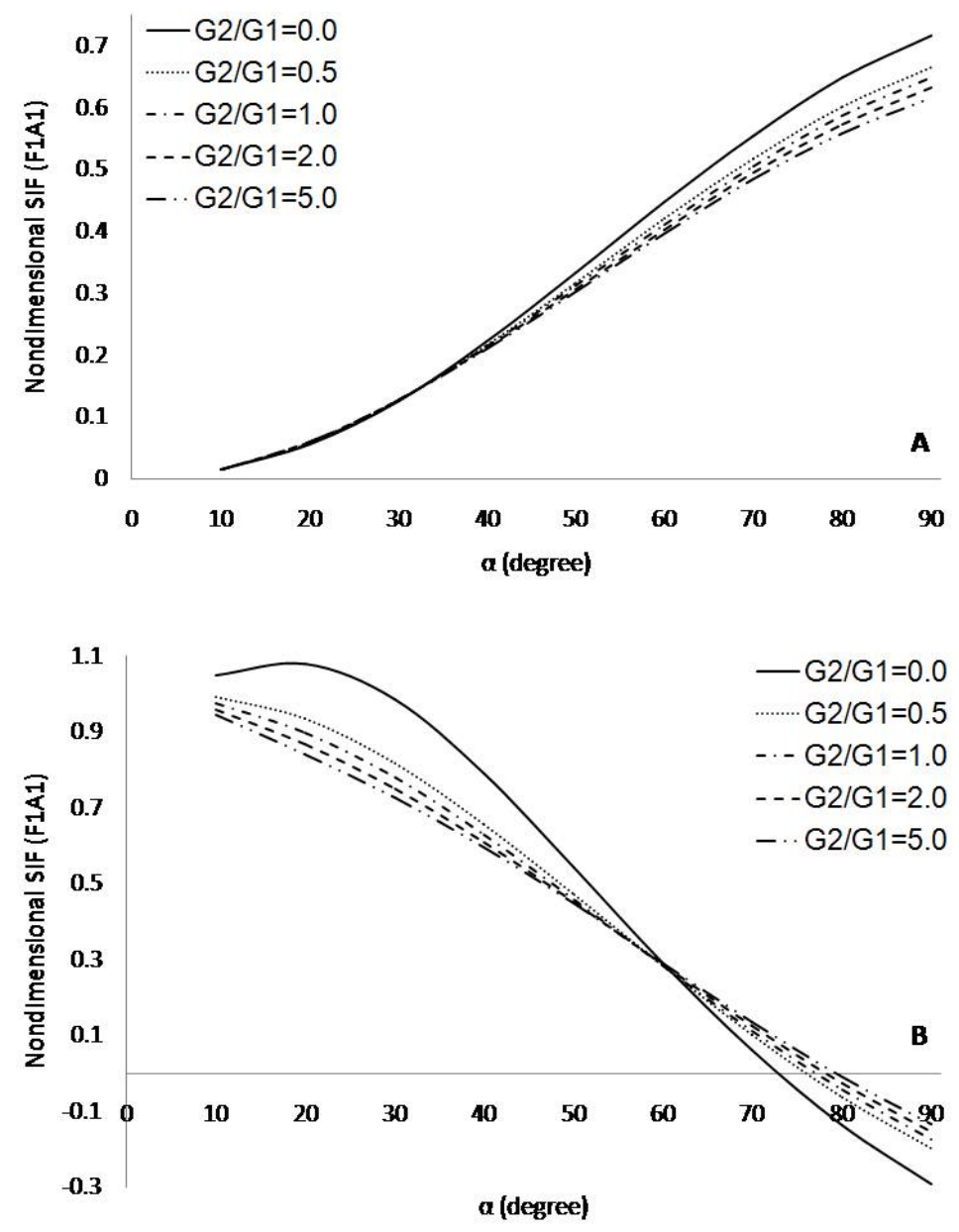

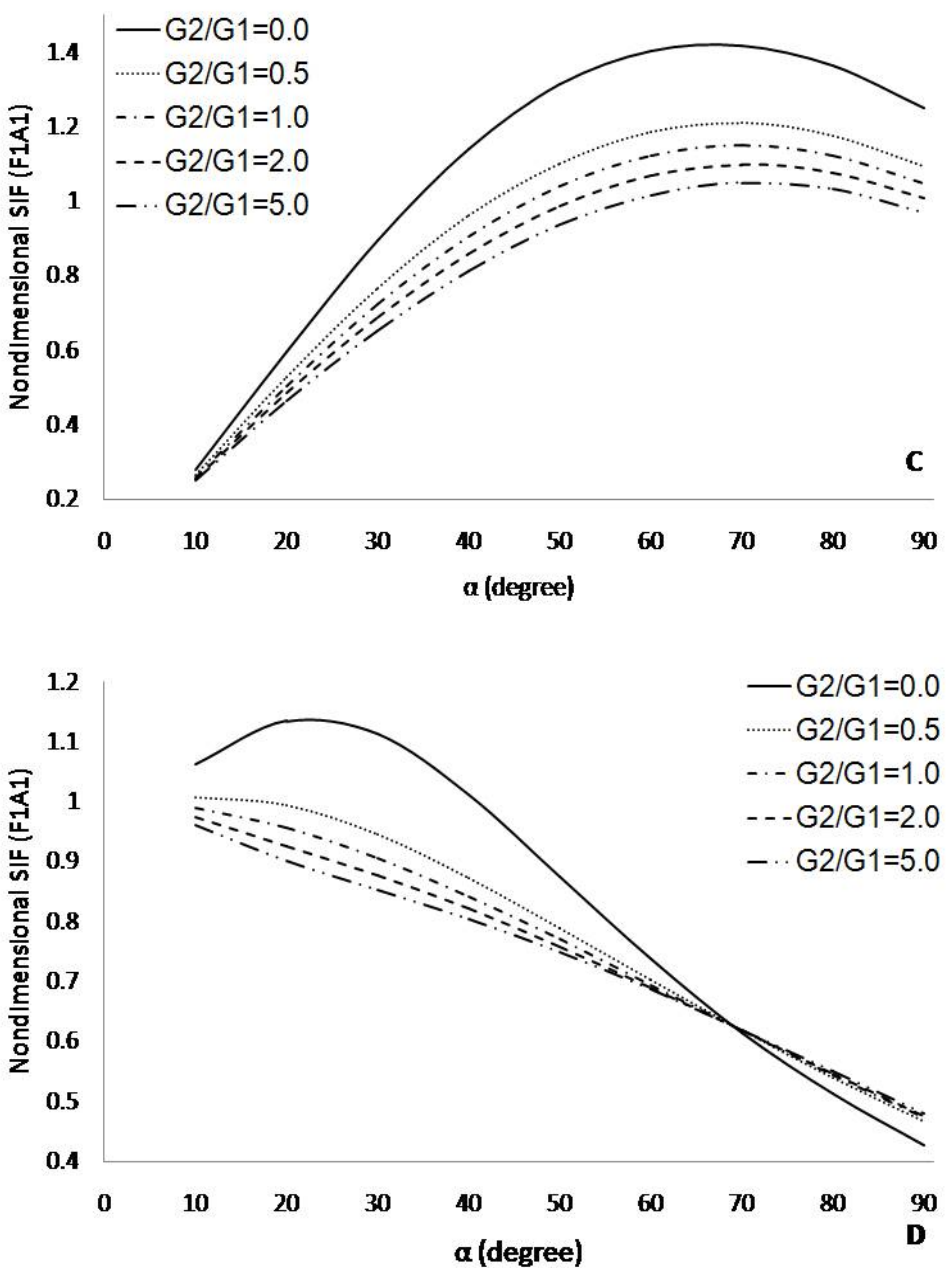

Figure 6. SIF for a circular arc crack when $h=0.5 R$ and $\alpha$ varies at crack tip $A_{l}$ subject to: Part A Shear stress, Part B Normal stress, Part C Tearing Stress and Part D Mixed stress

As $G_{2} / G_{1}$ increases the nondimensional SIF decreases subjected to shear stress $\sigma x_{1}=\sigma x_{2}=p$ and tearing stress $\sigma x_{1} y_{1}=\sigma x_{2} y_{2}=p$ at crack tip $A_{1}$. Whereas for normal stress $\sigma y_{1}=\sigma y_{2}=p$ the nondimensional SIF decreases when $\alpha<60^{\circ}$ and for mixed stress $\sigma x_{1}=\sigma x_{2}=\sigma y_{1}=\sigma y_{2}=p$ when $\alpha<70^{\circ}$ as $G_{2} / G_{1}$ increases at crack tip $A_{l}$.

\section{Conclusions}

In this paper, the HSIE for an inclined crack and a circular arc crack lies in the upper part of bonded dissimilar materials subjected to various remote stresses such as shear stress $\sigma x_{1}=\sigma x_{2}=p$, normal stress $\sigma y_{1}=\sigma y_{2}=p$, tearing stress $\quad \sigma x_{1} y_{1}=\sigma x_{2} y_{2}=p \quad$ or mixed stress $\sigma x_{1}=\sigma x_{2}=\sigma y_{1}=\sigma y_{2}=p$ are formulated by using the modified complex variable function method with the crack opening displacement function as the unknown function.
From the numerical results we conclude that the nondimensional SIF for a crack lies in the upper part of bonded dissimilar materials depends on the various remote stresses, the elastic constants ratio, the crack geometries and the distance between the crack and the boundary. The close the crack to the boundary the smaller the nondimensional SIF which interprets the weaker of the materials. However the nondimensional SIF is altered for different stresses, elastic constants ratio or crack geometries.

\section{Acknowledgements}

The first author would like to thanks the Ministry of Education Malaysia, Universiti Putra Malaysia and Universiti Teknikal Malaysia Melaka for the financial support. The second author would like to thank the Universiti Putra Malaysia for Putra Grant, project no: 9567900. We gratefully acknowledge financial support by Universiti Teknologi MARA (UiTM). 


\section{REFERENCES}

[1] N.M.A. Nik Long, M.R. Aridi, Z.K. Eshkuvatov. Mode stresses for the interaction between an inclined crack and a curved crack in plane elasticity, Mathematical Problems in Engineering, Vol. 2015, 1-10, 2015.

[2] R.A. Rafar, N.M.A. Nik Long, N. Senu, N.A. Noda. Stress intensity factor for multiple inclined or curved cracks problem in circular positions in plane elasticity, ZAMM Journal of Applied Mathematics and Mechanics, Vol.97, No.11, 1482-1494, 2017.

[3] Y.H. Chen. Solution of stiffened problems for a finite internally cracked plate by using complex potentials and the generalized variational method, Computer Methods in Applied Mechanics and Engineering, Vol.62, 1-16, 1987.

[4] J. Lai, J. Schijve. The stress intensity factor and stress concentration for a finite plate with a single crack emanating from a hole, Engineering Fracture Mechanics, Vol.36, No.4, 619-630, 1990.

[5] Y.Z. Chen, X.Y. Lin, X.Z. Wang. Numerical solution for curved crack problem in elastic half-plane using hypersingular integral equation, Philosophical Magazine, Vol.89, No.26, 2239-2253, 2009.

[6] N.R.F. Elfakhakhre, N.M.A. Nik Long, Z.K. Eshkuvatov. Stress intensity factor for an elastic half plane weakened by multiple curved cracks, Applied Mathematical Modelling, Vol.60, 540-551, 2018.

[7] M. Isida, H. Noguchi. Arbitrary array of cracks in bonded half planes subjected to various loadings, Engineering Fracture Mechanics, Vol.46, No.3, 365-380, 1993.

[8] Y.Z. Chen. Multiple crack problems for two bonded half planes in plane and antiplane elasticity, Engineering Fracture Mechanics, Vol.25, No.1, 1-9, 1986.

[9] Y.Z. Chen, N. Hasebe. Stress-intensity factors for curved circular crack in bonded dissimilar materials, Theoretical and Applied Fracture Mechanics, Vol.17, 189-196, 1992.

[10] M. Yang, X. Wang. A bridged crack perpendicular to a bimaterial interface, Acta Mechanica, Vol.229, No.5, 2063-2078, 2018.

[11] K. Miura, M. Sakamoto, K. Kobayashi, J.A. Pramudita, Y. Tanabe. An analytical solution for the axisymmetric problem of a penny-shaped crack in an elastic layer sandwiched between dissimilar materials, Mechanical Engineering Journal, Vol.5, No.3, 1-12, 2018.

[12] Z. Chen, Y. Xiong, H. Qiu, G. Lin, Z. Li. Stress intensity factor-based prediction of solidification crack growth during welding of high strength steel, Journal of Materials Processing Technology, Vol.252, 270-278, 2018.

[13] K. Huang, L. Guo, H. Yu. Investigation on mixed-mode dynamic stress intensity factors of an interface crack in bi-materials with an inclusion, Composite Structures, Vol.202, 491-499, 2018.

[14] N.I. Muskhelishvili, Some Basic Problems of the Mathematical Theory of Elasticity, Noordhoff International 